\title{
Wafer-scale and Full-coverage Two-dimensional Molecular Monolay- ers Strained by Solvent Surface Tension Balance
}

\author{
Baichuan Jiang, ${ }^{\dagger, \#}$ Yu Che, ${ }^{\dagger}, \#$ Yurong Chen, ${ }^{\dagger}$ Yingxuan Zhao, ${ }^{\dagger}$ Can Wang, ${ }^{\ddagger}$ Wenbin Li, $^{\dagger}$ Hongxian \\ Zheng, ${ }^{\dagger}$ Xinxin Huang, ${ }^{\dagger}$ Paolo Samorì, ${ }^{*}$ and Lei Zhang*,†
}

tKey Laboratory of Mesoscopic Chemistry of MOE, School of Chemistry and Chemical Engineering, Nanjing University, Nanjing, Jiangsu 210023, China

¥University of Strasbourg, CNRS, ISIS UMR 7006, 8 allée Gaspard Monge, F-67000 Strasbourg, France

KEYWORDS: two-dimensional organic materials, molecular monolayer, Langmuir-Blodgett technique, organic fieldeffect transistors, surface tension balance

\begin{abstract}
Inspired by the outstanding properties discovered in two-dimensional materials, the bottom-up generation of molecular monolayers is becoming again extremely popular as a route to develop novel functional materials and devices with tailored characteristics and minimal materials consumption. However, achieving a full-coverage over a large-area still represents a grand challenge. Here we report a molecular self-assembly protocol at the water surface in which the monolayers are strained by a novel solvent surface tension balance (SSTB) instead of a physical film balance as in the conventional LangmuirBlodgett (LB) method. The obtained molecular monolayers can be transferred onto any arbitrary substrate including rigid inorganic oxides and metals, as well as flexible polymeric dielectrics. As a proof-of-concept, their application as ideal modification layers of a dielectric support for high-performance organic field-effect transistors (OFETs) has been demonstrated. The field-effect mobilities of both $\mathrm{p}$ - and n-type semiconductors displayed dramatic improvements of 1-3 orders of magnitude on SSTB-derived molecular monolayer, reaching values as high as $6.16 \mathrm{~cm}^{2} \mathrm{~V}^{-1} \mathrm{~s}^{-1}$ and $0.68 \mathrm{~cm}^{2} \mathrm{~V}^{-1} \mathrm{~s}^{-1}$ for pentacene and PTCDI-C8, respectively. This methodology for the fabrication of wafer-scale and defect-free molecular monolayers holds potential towards the emergence of a new generation of high-performance electronics based on two-dimensional materials.
\end{abstract}

\section{INTRODUCTION}

The numerous ground-breaking studies casting fresh light onto the outstanding physical and chemical properties of graphene and other two-dimensional (2D) materials have revitalized the interest of the scientific community on molecular monolayers. ${ }^{1-4}$ Owing to the structural diversity of the molecules and their tunable electronic characteristics, 2D organic monolayers are considered to be attractive building blocks for novel applications in nano-electronics and opto-electronics. 5,6 Among them, self-assembled monolayers (SAMs) have been widely used as active elements in second-order non-linear optics, ${ }^{7}$ organic field-effect transistors (OFETs), ${ }^{8}$ light-emitting diodes, ${ }^{9}$ molecular tunneling/rectifying junctions, ${ }^{10}$ non-volatile memories, ${ }^{11}$ optoelectronics ${ }^{12}$ and organic electronics. ${ }^{13}$ The combination of molecular monolayers with inorganic 2D materials enables the fabrication of novel device prototypes based on organic/inorganic van der Waals heterojunctions (vdWHs). ${ }^{14,15}$ Furthermore, organic molecules pre-assembled into monolayers could also serve as precursors or soft templates for the synthesis of novel 2D polymers and covalent organic frameworks (COFs), which will further enrich the category of 2D materials at the disposal of nanotechnology. $16-18$
So far, the most commonly used methods for constructing molecular monolayers rely on the in-situ self-assembly at solid/vapor or solid/liquid interfaces. ${ }^{19-22}$ However, in such methods, the molecules and substrate surface must expose specific chemical functionalities which interact at the covalent or non-covalent level yielding chemisorption or physisorption, respectively. ${ }^{23}$ In some cases, the use of the simple spin-coating or dip-coating method can also generate ultrathin film or even molecular monolayers, provided the solution surface tension, solvent dielectric constant, solvent boiling point and molecular concentration are suitable..$^{24-26}$ However, the use of organic solvents during monolayer formation in spin-coating or dip-coating may potentially dissolve the underlying layer such as the semiconductor or dielectrics, thereby hindering the fabrication of multi-layered and multi-component structures. The formation of a molecular monolayer ex-situ followed by its transfer on the surface of interest represents viable route to overcome such limitations. The most popular method following such a strategy is the Langmuir-Blodgett (LB) technique which allows to assemble both amphiphilic and hydrophobic molecules. ${ }^{27-30}$ However, the sophisticated nature of the equipment and the slow nature of the processing makes this method a research tool for fundamental lab-scale studies rather than a powerful manufacturing technology. 
Against this backdrop, we have devised here a general method for the fabrication of large-area and full-coverage molecular monolayers with minimal structural defects. Toward this end, amphiphilic molecules were first pre-assembled at the air/water interface as in the LB technique. The freely-floating molecules were then compressed not using physical barriers but rather by dropping a low-surface tension organic solvent onto the water surface, in order to convert the partially-covering molecular monolayer into a fully-covering one (Figure 1a). Such compression process is driven by the surface tension differences between the organic solvent and water, and hence can be adjusted via the careful choice of the organic solvent. In addition, the 2D molecular monolayer can be transferred onto any arbitrary substrate regardless of its surface chemical properties and hence is not restricted to specific anchoring groups. The morphology of the obtained molecular monolayers was characterized by atomic force microscopy (AFM) imaging. The quality of the 2D amphiphilic monolayers was also assessed through the preparation and characterization of OFETs in which the semiconductor/dielectric interface was optimized via the incorporation of such molecular monolayers.

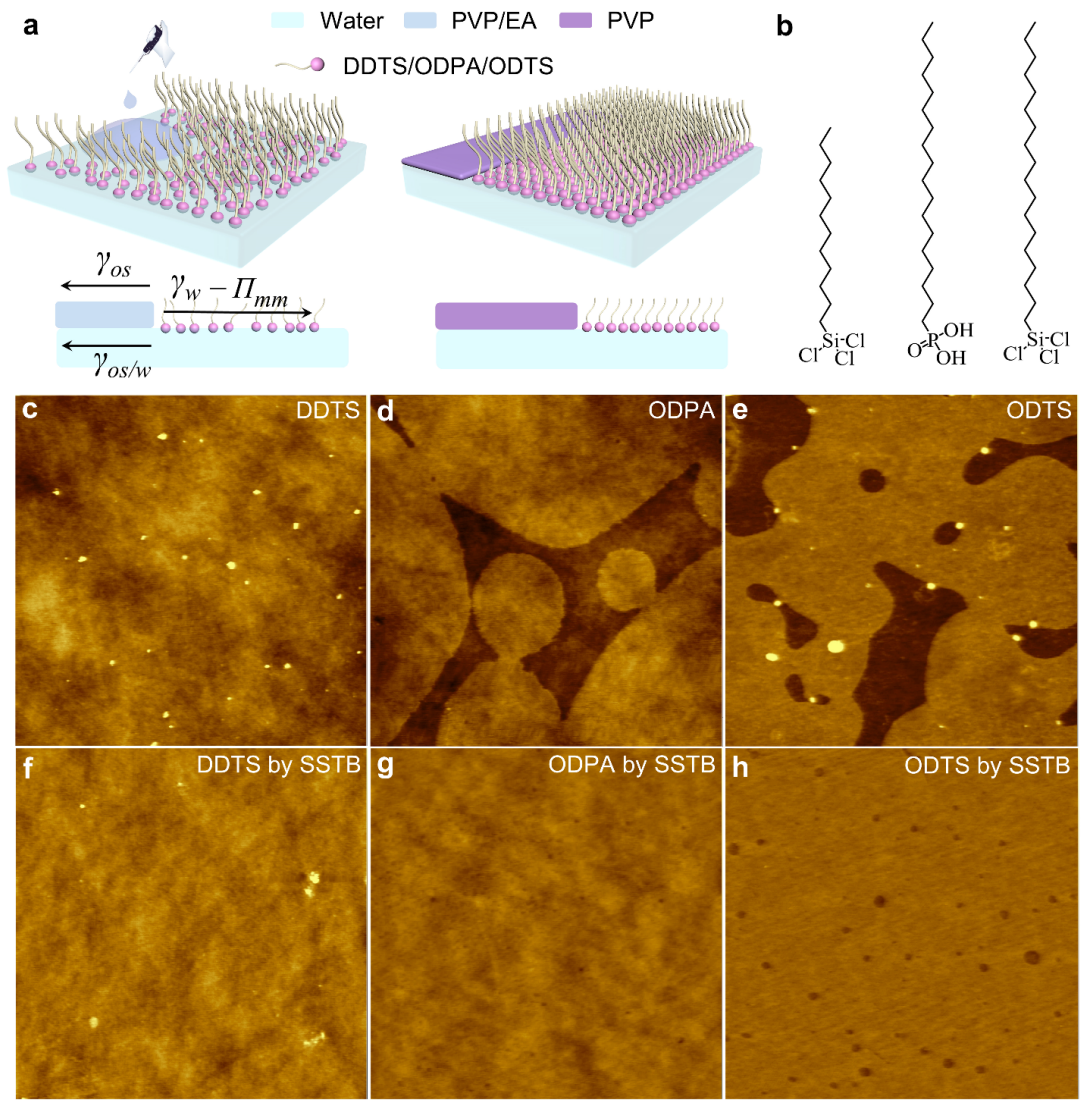

Figure 1. Schematic illustration of the solvent surface tension balance (SSTB) method and its applications in assembling 2D molecular monolayers: (a) Schematic diagram of the amphiphilic molecular monolayer floating on the water before (left) and after (right) compression by SSTB; (b) Chemical structures of dodecyltrichlorosilane (DDTS, left), octadecylphosphonic acid (ODPA, middle), octadecyltrichlorosilane (ODTS, right); (c-h) AFM images of different molecular monolayers on silicon wafer without (c-e) and with (f-h) compression by SSTB. Scan area $=5 \mu \mathrm{m} \times 5 \mu \mathrm{m}$. Z-scale $=6 \mathrm{~nm}$.

As proof-of-concept, three commercially available prototypical amphiphilic molecules, namely dodecyltrichlorosilane (DDTS), octadecyltrichlorosilane (ODTS), and octadecylphosphonic acid (ODPA), whose molecular structures are portrayed in Figure $1 \mathrm{~b}$, were chosen to demonstrate the experimental design presented herein. Among them, ODTS has been widely used to construct SAMs to render hydrophobic the silicon dioxide $\left(\mathrm{SiO}_{2}\right)$ surface, which has resulted in performance improvements in transistors based on organic semiconductors, ${ }^{24,29}$ graphene, ${ }^{31}$ and transition-metal dichalcogenides ${ }^{32}$ by also eliminating the charge carrier traps associated with the dangling bond on bare $\mathrm{SiO}_{2}$. Here, ODTS was also taken as an essential benchmark to evaluate the solvent surface tension balance (SSTB) method used to fabricate 2D molecular monolayers in this study. Firstly, ODTS molecules were spread uniformly onto the water surface to exclusively assemble into a single- layer. The $\mathrm{Si}-\mathrm{Cl}$ bond is extremely humidity-sensitive, thus ODTS molecules tend to undergo hydrolyzation followed by their cross-linking through dehydration condensation. In light of this, the yield for forming a monolayer had to be optimized according to the following criteria: 1) the solvent used to dissolve ODTS was anhydrous to avoid cross-linking reactions in solution; 2) the surface tension and boiling point of the chosen solvent was as low as possible to accelerate the spreading and drying process when the ODTS solution was dropped onto the water surface. Furthermore, the solvent dielectric constant $(k)$, the length of the amphiphilic chain and the amphiphile concentration were also taken into consideration to avoid the formation of micelles in solution, to avoid the formation of bi- or multi-layer at the air/water interface upon the evaporation of the organic solvent. 
After optimization, a "clean" monolayer was successfully obtained for all of the three amphiphilic molecules, as revealed by the AFM characterization (Figure 1c-e). Interestingly, these molecules showed distinctly different morphologies when transferred to the silicon wafer. In particular, DDTS exhibited a smooth morphology without an obvious layered structure (Figure 1c). It was speculated that the DDTS molecules were in the gas phase, as its molecular weight is the smallest among all the three amphiphilic molecules. Conversely, the ODPA films exhibited a partially-covering monolayer with round domains (Figure 1d), suggesting that the monolayer was in the liquid phase, thereby allowing it to migrate freely within the monolayer in order to minimize the system free energy. Due to the strong intermolecular interactions determined by both $\mathrm{Si}-\mathrm{OH}$ and long alkyl chains, the ODTS membrane floating on the water surface can be expected to be in the solid state, with layered structural features similar to ODPA, but with irregularlyshaped monolayer domains (Figure 1e).

To obtain a defect-free 2D monolayer, the molecules concentrated on the water surface were further constrained by the organic solvent (e.g., ethyl acetate, EA), resulting in enhanced packing density (Figure 1a). Owing to the much lower surface tension of EA compared to that of water (23.9 $\mathrm{mN} / \mathrm{m}$ vs $72.8 \mathrm{mN} / \mathrm{m}$ at $20{ }^{\circ} \mathrm{C}$ ), it spreads rapidly when dropped onto the water surface in order to minimize the total surface free energy. As a result, the surface area of the water, which corresponds to that of the molecular monolayer, shrinks until the molecular membrane tension increases and becomes equal to the surface tension difference between water and the organic solvent. In order to prevent the molecules in the monolayer from disassembling upon evaporation of the organic solvent, a small amount of polymer was added to the solvent (e.g., PVP for EA) to fill the surface when the organic solvent evaporates by occupying the area initially filled with solvent molecules before volatilization. Sudan Red dye could also be added to the organic solvent by mixing it with the polymer to enable better visualization of the boundary between the polymer and the monolayer (see Figure S1).

It is worth noting that the presence of excess droplets of polymer solution on the ODTS or ODPA monolayers does not cause further reduction of the monolayer surface area, because the surface area is ruled only by the surface energy balance between water, the organic solvent, and the monomolecular membrane. In contrast, the DDTS monolayer would continue to collapse upon addition of more EA, as amphiphilic molecules with shorter alkyl chains tend to form monolayers with a lower threshold tension to breakdown. Based on this observation, the surface areas of EAstrained ODTS monolayers were measured and plotted against the amount of ODTS molecules being pre-assembled (Figure S1c). As a result, a single molecule was estimated to occupy an area of $18.75 \AA^{2}$ in an ODTS monolayer being strained by PVP/EA. According to a previously published membrane pressure/area ( $\pi-A)$ curve for ODTS LB film, ${ }^{29}$ such a monomolecular area corresponds to a membrane pressure of $\sim 45.2 \mathrm{mN} / \mathrm{m}$, which almost perfectly matches the difference of $48.9 \mathrm{mN} / \mathrm{m}$ between the surface tensions of EA and water.

For DDTS and ODPA, it was found that both of them formed defect-free monolayers through the SSTB method, as evidenced by AFM imaging (Figure 1f,g). However, for ODTS, after EA/PVP compression some unwanted large holes/defects remained dispersed in the monolayer (Figure S2a). This phenomenon may be attributed to the different nature of the intermolecular interactions (i.e., gaseous, liquid, and solid) in the three solvents, as mentioned previously. Based on this hypothesis, two methods may be used to eliminate the holes in the ODTS monolayer: 1) using heat to "melt" the monolayer at elevated temperatures (e.g., 80 $\left.{ }^{\circ} \mathrm{C}\right)$ so that the ODTS molecules at the water surface behave more like a $2 \mathrm{D}$ gas/liquid phase rather than a solid state; 2 ) use a small molecular solvent as processing additive to enhance the plasticity of the monolayer upon compression. Although the former method is able to yield a very smooth and defect-free monolayer, as confirmed by AFM imaging (Figure S2b), it should be noted that the use of high temperatures will be accompanied by a greater degree of disorder among the alkyl chains, which could jeopardize the performance of OFETs fabricated using ODTS as modification layer at the dielectric/semiconductor interface (Figure S2d,e). As for the latter method, a series of common organic solvents were added to the subphase (i.e., water) to mitigate the intermolecular interactions of ODTS, among which it was found that acetone was the best choice to obtain a fullcoverage molecular monolayer of ODTS (Figure $1 \mathrm{~h}$ and Figure S2c).

Compared to conventional SAM modification methods such as liquid phase immersion (LPI) and vapor fumigation, ${ }^{33,34}$ which are restricted to specific types of chemical bonding between the molecules and the substrate, both the SSTB method and LB technique share a similar advantage in that the monolayer at the water/air interface can be transferred onto different substrates regardless of the surface chemistry. In this study, a full-coverage ODTS monolayer was successfully transferred to a variety of substrates including thermally-grown $\mathrm{SiO}_{2}, \mathrm{Al}_{2} \mathrm{O}_{3}$ obtained by in-situ oxidation, E-beam evaporated $\mathrm{SiO}_{2}$ and $\mathrm{Si}_{3} \mathrm{~N}_{4}$, some spincoated dielectric polymers such as cross-linked poly4-vinylphenol (PVP), polyacrylonitrile (PAN), and polyvinyl alcohol (PVA), and a representative inert metal (gold), as shown in Figure S3. The thickness of the 2D ODTS monolayer was $\sim 1.8 \mathrm{~nm}$ on all those films, in agreement with theoretical models of the molecular contour length and previous reports. ${ }^{29}$ Following the acid/base vapor-catalyzed polymerization of Si-OH, the ODTS monolayer exhibited sufficient stability to enable further rinsing and sonication in solvent, even on top of PAN, which does not possess any anchoring groups for ODTS. The potential to build stable molecular monolayers on top of arbitrary surfaces is expected to open up new applications in nano(opto-)electronics.

Currently, OFETs are key electronic components for the fabrication of bendable and stretchable smart circuitries. ${ }^{35}$ For a given organic semiconductor (OSC), the electrical performance of the resulting OFETs largely depends on the key interface between the OSC and the dielectrics. ${ }^{23}$ However, as dielectric materials are chosen mostly in view of their excellent dielectric strength, processability for film forming, stability upon solvent corrosion, and large dielectric constant, yet the dielectric layer used in OFETs cannot always form a high-quality interface with the OSC. In such cases, a modification layer is needed to take advantage of the intrinsic high mobility of the OSC. To evaluate the quality of SSTB-derived 2D molecular monolayers used as modification layers, 
bottom-gate top-contact OFETs were fabricated making use of various polymeric and inorganic dielectric films, which may

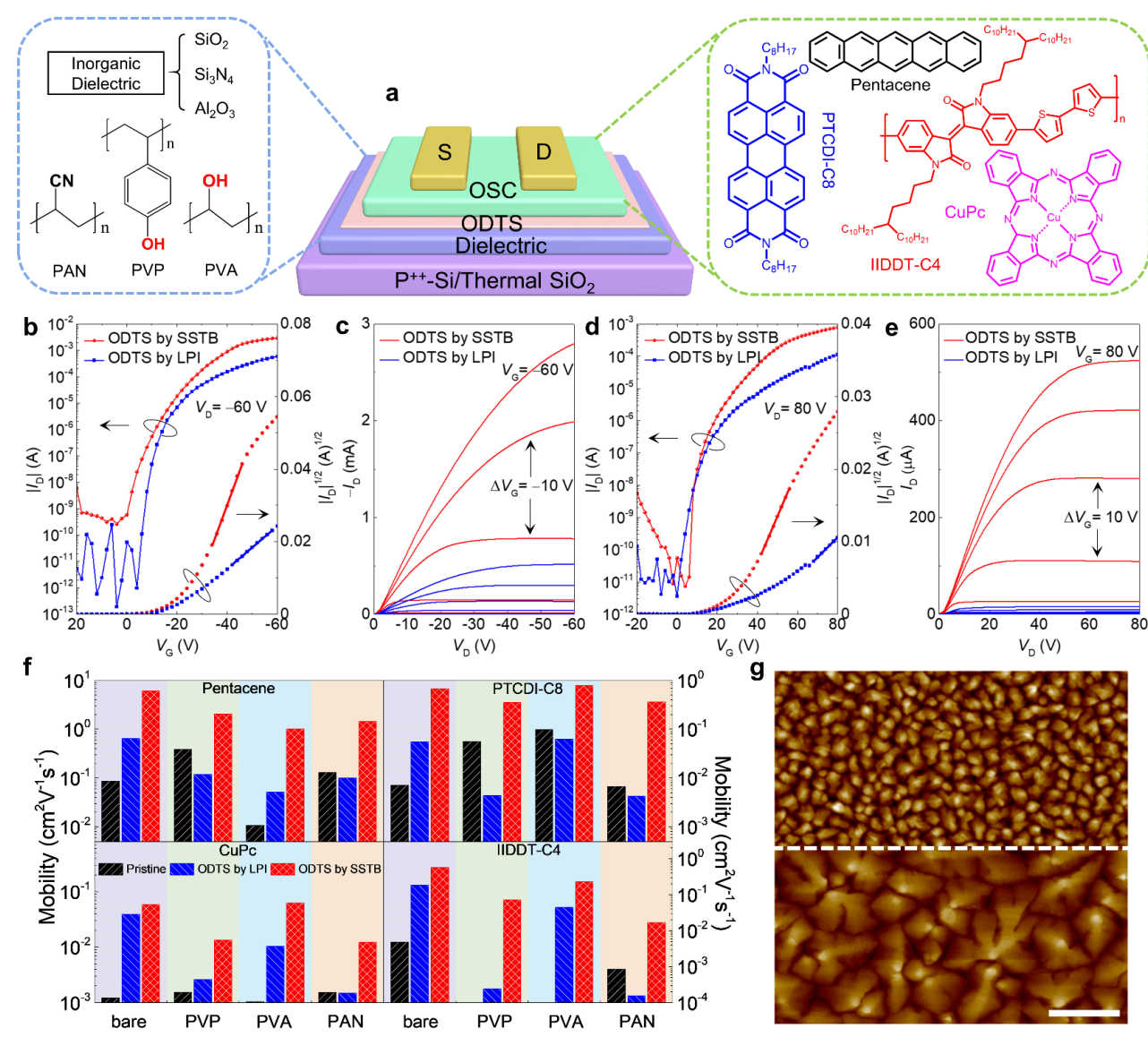

Figure 2. (a) Device structure of a bottom-gate top-contact organic field-effect transistor (OFET), and chemical structures of the gate-dielectric materials and organic semiconductor materials used therein. (b-e) Transfer $\left(I_{\mathrm{D}}-V_{\mathrm{G}}\right)$ and output $\left(I_{\mathrm{D}}-V_{\mathrm{D}}\right)$ characteristics of pentacene (b, c) and PTCDI-C8 (d, e) OFETs prepared with different ODTS monolayers on an $\mathrm{SiO}_{2} / \mathrm{Si}_{\text {dielectric layer. The red and }}$ blue curves represent ODTS prepared using SSTB and LPI, respectively. (f) Field-effect mobilities of various OFETs fabricated with different organic semiconductors and with different ODTS monolayers on different organic polymer dielectric layers. (g) AFM images of a $40 \mathrm{~nm}$ thick pentacene layer deposited on the $\mathrm{SiO}_{2} / \mathrm{Si}$ dielectric layer with ODTS from LPI (top panel) and SSTB (bottom panel) modification. Z-scales $=50 \mathrm{~nm}$. Scale bar $=1 \mu \mathrm{m}$.

be passivated through the transfer of a defect-free ODTS monolayer on top (Figure 2a). The values of thickness $(d)$, dielectric constant $(k)$, capacitance per unit area $\left(C_{i}\right)$ and root mean square roughness $(R M S)$ of these dielectric layers are reported in Table S1. The electrical performances of four common organic semiconductors were tested in such OFETs and their chemical structures are given in Figure 2a. Details of the device fabrication and measurement can be found in the experimental section in the Supporting Information (Figure S4-6, Table S2-5).

Following surface modification on various dielectric substrates, all the bottom-gate top-contact devices exhibited well-defined FET characteristics (Figure 2b-e). The doubleslope effect was observed here, however, by far we have not arrived consensus on understanding the physical principles behind this effect, which means that we can take some strategies, such as reducing contact resistance, to reduce or mitigate the nonideal behaviors in OFETs and further improve OFETs performance in the future. ${ }^{36}$ The key OFET parameters for the pentacene and PTCDI-C8 OSCs are summarized in Table 1. It is evident that the charge carrier mobilities of both $\mathrm{p}$ - and n-type semiconductors showed dramatic improvements of several orders of magnitude following ODTS-monolayer modification. For example, the mobility of pentacene on the $\mathrm{Al}_{2} \mathrm{O}_{3}$ surface improved by a factor of $\sim 280$, from $0.003 \mathrm{~cm}^{2} \mathrm{~V}^{-1} \mathrm{~s}^{-1}$ to $0.85 \mathrm{~cm}^{2} \mathrm{~V}^{-1} \mathrm{~s}^{-1}$. Further, as n-type OSCs are more sensitive to interfacial traps, PTCDIC8 exhibited an over 300-fold increase in its mobility when the inorganic dielectrics (e.g., $\mathrm{SiO}_{2}$ and $\mathrm{Si}_{3} \mathrm{~N}_{4}$ ) were passivated by the ODTS-monolayer. As for high- $k$ polymeric dielectrics, the OFETs mobilities and $I_{\text {on }} / I_{\text {off }}$ ratios also benefited markedly from the modification of the 2D molecular monolayer. For example, in a pentacene-based OFET, the modification of a polymer dielectric PAN with an ODTSmonolayer was accompanied with a 11-fold enhancement in the field-effect mobilities (from $0.129 \mathrm{~cm}^{2} \mathrm{~V}^{-1} \mathrm{~s}^{-1}$ to 1.44 $\mathrm{cm}^{2} \mathrm{~V}^{-1} \mathrm{~s}^{-1}$ ) and an increase of two orders of magnitude (from $10^{4}$ to $10^{6}$ ) of the $I_{\text {on }} / I_{\text {off }}$ ratio (Table 1 and Figure $S 4 \mathrm{c}$ ). Meanwhile, the mobilities of PTCDI-C8-based OFETs increased by a factor of 51 (from $0.007 \mathrm{~cm}^{2} \mathrm{~V}^{-1} \mathrm{~s}^{-1}$ to $0.36 \mathrm{~cm}^{2}$ $\mathrm{V}^{-1} \mathrm{~s}^{-1}$ ), and the $I_{\mathrm{on}} / I_{\text {off }}$ ratio increased by one orders of magnitude (from $10^{6}$ to $10^{7}$ ) (Table 1 and Figure S5c). Furthermore, the threshold voltage $\left(V_{\mathrm{T}}\right)$ consistently shifted to a more positive direction in a certain range for both $n$ - and $p$ - 
type small-molecule semiconductors regardless of the insulator used (Table S2-4), indicating the existence of dipole moments in the monolayer, which originate from the molecular orientation and ordering in 2D monolayers assembled at the water surface.

Table 1. Detailed device parameters of pentacene- and PTCDI-C8-based OFETs with different dielectric layers before and after modification with ODTS made using the SSTB method.

\begin{tabular}{|c|c|c|c|c|c|c|c|c|c|c|c|c|}
\hline \multirow{3}{*}{ Dielectric } & \multicolumn{4}{|c|}{ Mobility $\left(\mathrm{cm}^{2} \mathrm{~V}^{-1} \mathrm{~s}^{-1}\right)$} & \multicolumn{4}{|c|}{ Threshold voltage (V) } & \multicolumn{4}{|c|}{ On/off ratio } \\
\hline & \multicolumn{2}{|c|}{ pentacene } & \multicolumn{2}{|c|}{ PTCDI-C8 } & \multicolumn{2}{|c|}{ pentacene } & \multicolumn{2}{|c|}{ PTCDI-C8 } & \multicolumn{2}{|c|}{ pentacene } & \multicolumn{2}{|c|}{ PTCDI-C8 } \\
\hline & before & after & before & after & before & after & before & after & before & after & before & after \\
\hline $\mathrm{SiO}_{2}{ }^{a}$ & 0.087 & 6.16 & 0.007 & 0.68 & -24 & -24 & 20 & 28 & $10^{6}$ & $10^{7}$ & $10^{6}$ & $10^{8}$ \\
\hline PVP & 0.386 & 2.07 & 0.056 & 0.35 & -21 & -17 & 25 & 47 & 108 & $10^{6}$ & $10^{6}$ & $10^{7}$ \\
\hline PVA & 0.011 & 1.00 & 0.099 & 0.78 & -32 & -3 & 20 & 27 & $10^{7}$ & $10^{4}$ & $10^{6}$ & $10^{7}$ \\
\hline PAN & 0.129 & 1.44 & 0.007 & 0.36 & -21 & -18 & 16 & 28 & $10^{4}$ & $10^{6}$ & $10^{6}$ & $10^{7}$ \\
\hline $\mathrm{SiO}_{2} b$ & 0.030 & 2.32 & 0.001 & 0.33 & -22 & -11 & 14 & 19 & $10^{5}$ & $10^{8}$ & $10^{5}$ & $10^{8}$ \\
\hline $\mathrm{Si}_{3} \mathrm{~N}_{4}$ & 0.033 & 2.42 & 0.001 & 0.35 & -22 & -14 & 17 & 20 & $10^{4}$ & $10^{8}$ & $10^{6}$ & $10^{8}$ \\
\hline $\mathrm{Al}_{2} \mathrm{O}_{3}$ & 0.003 & 0.85 & $x$ & 0.13 & -2 & -23 & $x$ & 33 & $10^{3}$ & $10^{8}$ & $x$ & $10^{7}$ \\
\hline
\end{tabular}

a) Here the $300 \mathrm{~nm}$ native $\mathrm{SiO}_{2}$ on silicon wafer was grown by thermal dry oxidation; b) Such a thin interlayer of $30 \mathrm{~nm}$ SiO was deposited by E-beam evaporation.

In previous studies, ODTS SAM was prepared mainly by LPI. ${ }^{19,34}$ Hence, LPI-derived ODTS-SAMs were also fabricated and tested in the same OFET configurations as a control experiment, with typical transfer and output characteristics as in Figure 2b-e. Not surprisingly, ODTS molecules only aid in the device performance by forming SAM if the dielectric materials possess abundant $\mathrm{C}-\mathrm{OH}$ or $\mathrm{Si}-\mathrm{OH}$ groups such as in the case of $\mathrm{SiO}_{2}$, PVP, and PVA, but not that of PAN (Figure 2f). Although the ODTS monolayers produced by the two different methods showed comparable surface roughness and exhibited smooth morphologies (Figure S7), it was found that the OFETs with a dielectric layer modified by means of the SSTB technique possessed much better device performance than those obtained using the conventional
LPI method (Figure 2f). For example, the average mobilities of pentacene OFETs based on SSTB-ODTS-monolayer and LPI-ODTS-SAM were determined to be $6.16 \mathrm{~cm}^{2} \mathrm{~V}^{-1} \mathrm{~s}^{-1}$ and $0.64 \mathrm{~cm}^{2} \mathrm{~V}^{-1} \mathrm{~s}^{-1}$, respectively, which represents one of the best results for pentacene-based top contact OFETs (Table S6). Such a pronounced performance difference may be attributed to the much larger crystalline grains (1.3 microns vs $300 \mathrm{~nm}$, as revealed by AFM imaging) in pentacene films grown on the SSTB-ODTS-monolayer modified $\mathrm{SiO}_{2}$ (Figure $2 \mathrm{~g})$. In vacuum-grown polycrystalline OSCs films, the presence of small crystalline grains indicates high nucleation density, which typically originates from the uniformity of the surface energy and so the structural disorders in the LPI-monolayer in this case. 

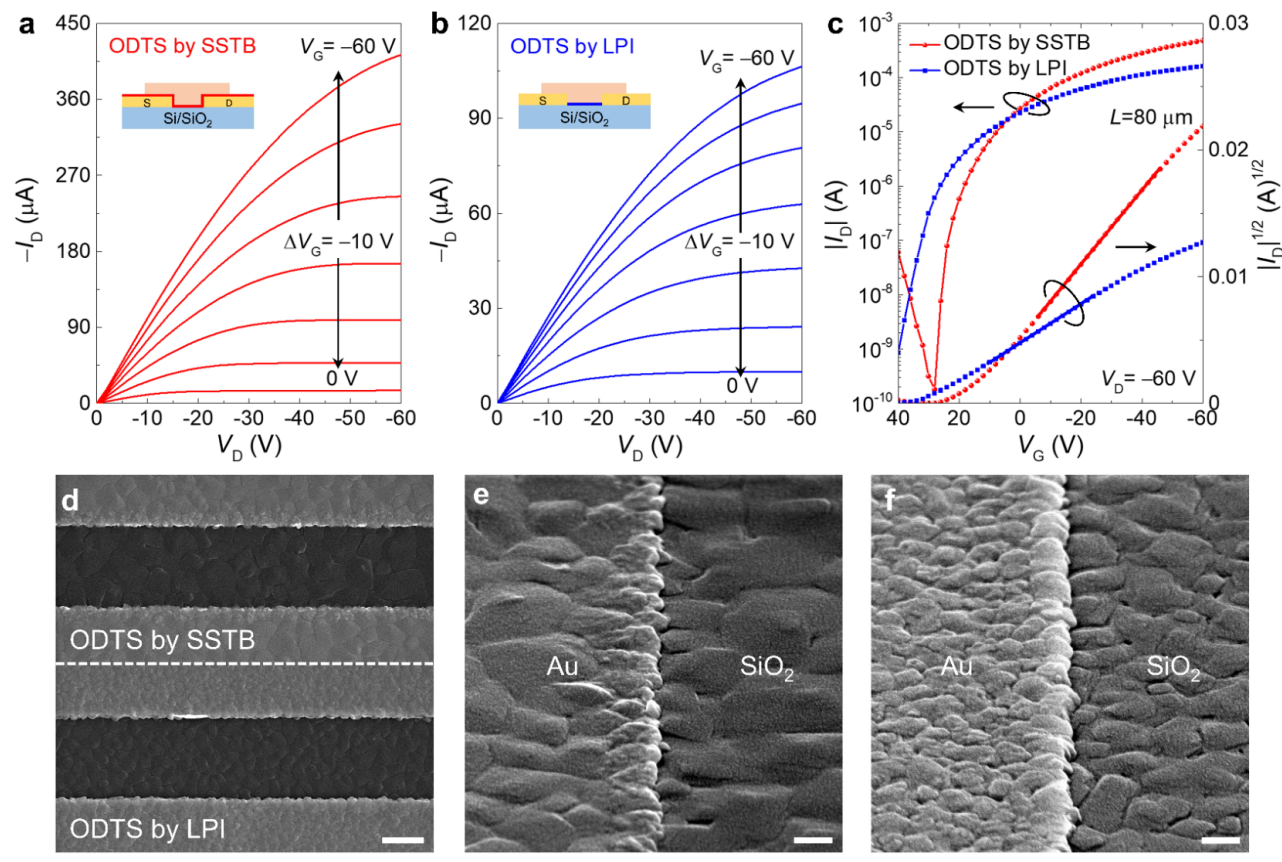

Figure 3. $(\mathrm{a}-\mathrm{c})$ Transfer $\left(I_{\mathrm{D}}-V_{\mathrm{G}}\right)$ and output $\left(I_{\mathrm{D}}-V_{\mathrm{D}}\right)$ characteristics of bottom-gate bottom-contact pentacene OFETs prepared with ODTS made using the SSTB and LPI methods on $\mathrm{SiO}_{2} / \mathrm{Si}$ substrate. The red and blue curves represent ODTS from SSTB and ODTS, respectively. (d) SEM images of a $40 \mathrm{~nm}$ thick pentacene layer deposited on $\mathrm{Au}$ and $\mathrm{SiO}_{2}$ substrates with ODTS from SSTB (top panel) and ODTS by LPI (bottom panel) modification. (e, f) Zoomed-in SEM images of the samples in (d) (top and bottom panels, respectively) with $60^{\circ}$ substrate tilt angle. Scale bars: (d) $1 \mu \mathrm{m}$; (e, f) $200 \mathrm{~nm}$.

In order to optimize single-molecular membranes as modification layers, it is necessary to unravel the relationship between the nature of the molecular monolayer, the morphology of OSC, and the electrical performance of corresponding devices. Hence, pentacene was deposited on $\mathrm{SiO}_{2}$ modified by the monolayers of uncross-linked ODTS, ODPA, and DDTS, respectively. AFM characterization was performed to monitor the surface topography of the pentacene thin films deposited on these molecular monolayers. The resulting morphological images were then correlated with the electrical performance as determined by OFET measurements (Figures S8 and S9). Unlike the LPI-ODTS modified surface, pentacene tends to grow into large crystal grains with sizes of $0.9-3.2 \mu \mathrm{m}$ which are similar to those of cross-linked ODTS molecular monolayers which have been strained by the EA/PVP solution. However, the charge carrier mobilities of OFETs with the aforementioned modification layers were quite different and are ordered as follows: cross-linked ODTS $\left(6.16 \mathrm{~cm}^{2} \mathrm{~V}^{-1} \mathrm{~s}^{-1}\right)>$ cross-linked DDTS $\left(3.23 \mathrm{~cm}^{2} \mathrm{~V}^{-1} \mathrm{~s}^{-1}\right)>$ uncross-linked ODTS $\left(2.42 \mathrm{~cm}^{2} \mathrm{~V}^{-1} \mathrm{~s}^{-1}\right)$ $>$ ODPA (1.54 $\left.\mathrm{cm}^{2} \mathrm{~V}^{-1} \mathrm{~s}^{-1}\right)$. Due to the key semiconducting channel, which consists of the one to two layers of pentacene adjacent to the dielectric surface, is in close contact with the identical chemical groups in the alkyl chains of the modified OFETs with amphiphilic monolayers, the pronounced performance differences can be explained only through the existence of remote interference exerted by the polarization and charge-traps underneath alkyl chains on the charge carrier transport.
In contrast with the conventional self-assembling process, which relies on specific surface groups and chemical bonds, one advantage of the SSTB method lies in its substrate tolerance. For example, even the gold surface can be modified with silane molecules like ODTS by using this method (Figure S10). This capability also makes the SSTB method suitable for the modification of OFETs in a bottomcontact configuration, in which the source and drain electrodes are pre-patterned at high-resolutions via photolithography, ink-jet printing, or electron beam lithography (EBL). As illustrated in Figure 3a, the modification of the bottom electrodes and the dielectric layer is performed simultaneously by transferring the ODTS monolayer on top. The output and transfer curves of bottom-contact OFETs modified by LPI-ODTS-SAMs and SSTB monolayer are compared in Figure 3a-c, respectively. After simultaneous modification of both electrode/OSC and OSC/insulator key interfaces by the ODTS-monolayer, the mobility of OFETs reached $1.02 \mathrm{~cm}^{2} \mathrm{~V}^{-1} \mathrm{~s}^{-1}$, which represents one of the best results for pentacene-based bottom contact OFETs. As shown in the SEM images (Figure 3d-f) and corresponding AFM images (Figure S11b,c), pentacene grows into larger crystalline domains on a gold electrode passivated by an SSTB-modification monolayer.

Essentially, SSTB-derived 2D molecular monolayers are compatible with large-area and flexible substrates. As shown in the photograph in Figure 4a, a solvent-strained ODTS monolayer with surface area exceeding $100 \mathrm{~cm}^{2}$ was pre-assembled at the water/air interface and successfully transferred onto a 3 inches $(76.2 \mathrm{~mm}$ ) silicon wafer. A $9 \times 9$ 
array of OFETs was then fabricated by depositing pentacene thin film and gold source-drain electrodes in sequence, with the typical transfer curves illustrated in Figure S12a. As shown in Figure 4c, the charge carrier mobility of 81 devices was in the range of $0.75-1.74 \mathrm{~cm}^{2} \mathrm{~V}^{-1} \mathrm{~s}^{-1}$, with an average value of $1.22 \mathrm{~cm}^{2} \mathrm{~V}^{-1} \mathrm{~s}^{-1}$. The OFETs could serve as indicators enabling to quantify the quality of the modification layer, and their narrow performance distribution in an array suggests that the ODTS monolayer was indeed fullycovering and quite uniformly the wafer-scale substrate. Since there is no limitation on the container size for the use

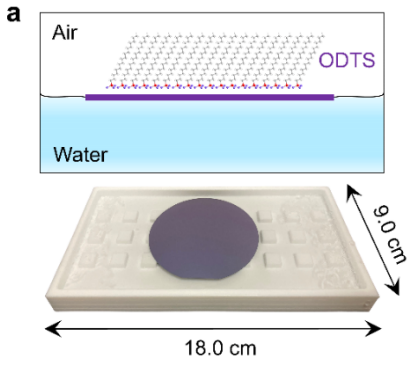

d

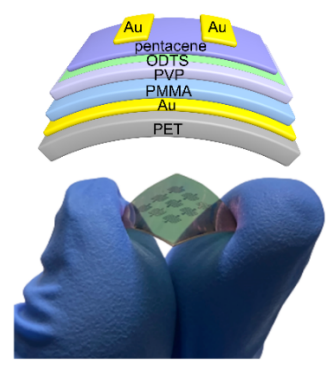

b
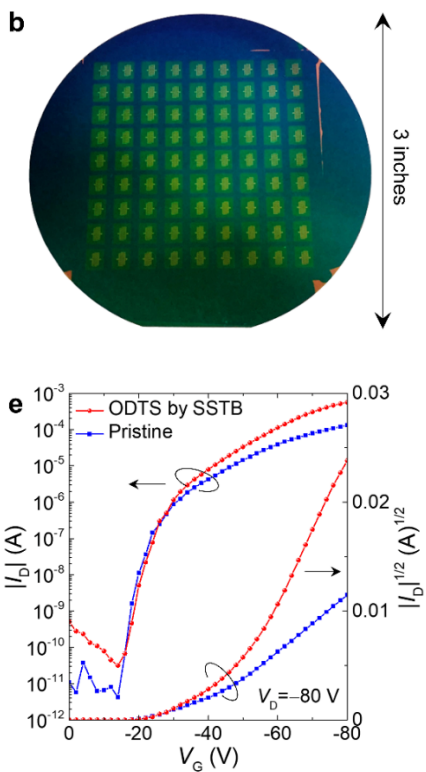
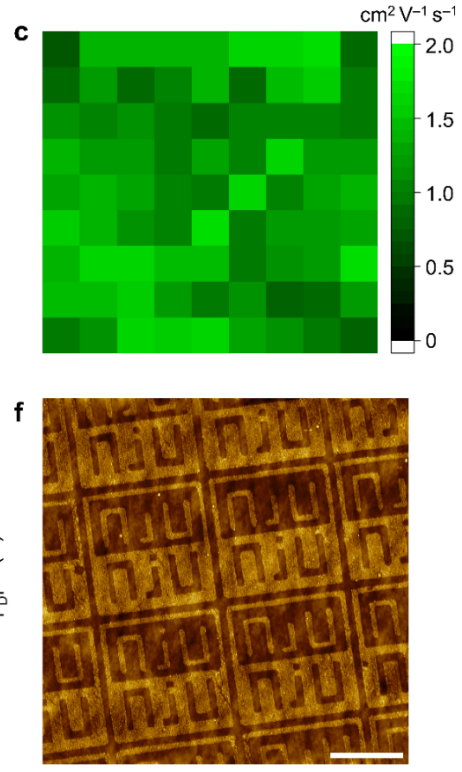

Figure 4. (a) Photograph of the apparatus used for transferring ODTS monolayer on the water surface onto a 3 inches (76.2 mm) silicon wafer with a $500 \mathrm{~nm}$ thermally grown $\mathrm{SiO}_{2}$ surface. (b) Photograph of wafer-scale OFET array. (c) Distribution of device mobilities. (d) Schematic structure (above) and photographic image (below) of the OFET array. (e) Typical transfer curves of flexible pentacene OFETs fabricated with PMMA/PVP dielectric layer modified with ODTS made using the SSTB method. (f) AFM image of a high-resolution ODTS “NJU” pattern prepared by PDMS stamp printing. Scale bar $=10 \mu \mathrm{m}$. Z-scale =12 $\mathrm{nm}$.

of organic solvents as compressing agents, the SSTB method can be conveniently applied at even larger scales than those demonstrated in this study, and thus has the potential to make single-molecule coatings more appealing in industry.

Another benefit of high-quality molecular monolayer modification can be obtained through the optimization of the performance of flexible electronic devices. To demonstrate this, a prototype device was fabricated by depositing the ODTS monolayer onto a composite dielectric layer comprised of polymeric substrate of PET and PVP/PMMA using the SSTB method. ${ }^{37}$ A schematic of the device configuration and a photograph of the flexible OFETs is shown in Figure $4 \mathrm{~d}$. It was found that the incorporation of the ODTS monolayer as the interfacial layer between PVP and pentacene enhanced the OFET mobility by a factor of 6.3 , reaching an average value of $1.03 \mathrm{~cm}^{2} \mathrm{~V}^{-1} \mathrm{~s}^{-1}$ (Figure 4e). The stability of the ODTS monolayer on polymers of PVA and PAN was characterized by AFM, revealing that the layered structure does not change during a storage period exceeding 2 months (Figure S13). Interestingly, the ODTS monolayer floating on the water surface can also be transferred to a hydrophobic PDMS silicon stamp with alkyl chains and Si-OH groups pointing inwards and outwards, respectively, for further transferring and high-resolution patterning (Figure $4 \mathrm{f}$ and Figure S14c). ${ }^{38}$

\section{CONCLUSION}

In summary, this study has showcased a general method for the preparation of wafer-scale and full-coverage molecular monolayers with the aid of the surface tension balance between water and various organic solvents. This radically new method holds the potential to replace conventional LB techniques and opens perspectives towards its wide application of 2D organic monolayers. The obtained molecular monolayers may be readily transferred either to dielectric layer and metal electrodes as a modification layer for highperformance flexible OFETs, or to PDMS soft stamps for the further micro-contact printing of high-resolution patterns, which can also be used for depositing molecular monolayers onto humidity-sensitive substrates. The performance of OFETs with novel device configurations such as vertical channel structure could also benefit from a high-quality and dense molecular monolayer passivating the nanoelectrodes, as demonstrated in this study where the charge carrier mobility of bottom-contact OFETs has displayed an over 10 -fold times improvement from $0.10 \mathrm{~cm}^{2} \mathrm{~V}^{-1} \mathrm{~s}^{-1}$ to $1.02 \mathrm{~cm}^{2} \mathrm{~V}^{-1} \mathrm{~s}^{-1}$ before and after ODTS monolayer modification. In the future, it may be possible to design and assemble many other functional molecules into monolayers as well, thereby increasing the availability of tightly packed molecular monolayers in both academic and industrial labs that are not equipped with LB instruments. Furthermore, the existence of transferrable and defect-free organic 2D materials will pave the way towards the construction of novel nano heterostructures and superlattices, in which both the structure and orientation of molecules may be designed and determined through rational molecular engineering.

\section{EXPERIMENTAL SECTION}

Materials. Dodecyltrichlorosilane (DDTS, 95\%), octadecyltrichlorosilane (ODTS, 90\%), octadecylphosphonic acid (ODPA, 97\%) were all purchased from Sigma-Aldrich and 
used as received without further purification. Pentacene (99.9\%), CuPc (99\%) and PTCDI-C8 (98\%) were purchased from Sigma-Aldrich and purified twice, twice and thrice by zone sublimation before usage, respectively. IIDDT-C4 was purchased form 1-Material and used without further purification. The polyethylene terephthalate (PET) substrate was provided by Nanjing XFNANO Materials Tech Co., Ltd, China.

Preparation of Various Dielectric Layers. For organic polymer dielectric layers, poly(vinyl alcohol) (PVA, Aldrich, number-average molecular weight; $M_{\mathrm{w}}=146,000 \sim 186,000$ ) solution (15 mg/ml) was prepared in de-ionized water. The PVA solution was spin-coated onto cleaned $\mathrm{SiO}_{2} / \mathrm{Si}$ substrate at $3000 \mathrm{rpm}$ for $40 \mathrm{~s}$. The coated films were crosslinked by annealing at $100{ }^{\circ} \mathrm{C}$ for $1 \mathrm{~h}$ and then $150{ }^{\circ} \mathrm{C}$ for 2 $\mathrm{h}$ on hot plate in an air atmosphere. $20 \mathrm{mg} / \mathrm{ml}$ poly(4-vinylphenol) (PVP, Aldrich, $\left.M_{\mathrm{w}}=25,000\right)$ solution was prepared by using poly(melamine-co-formaldehyde) methylated (PMF, Aldrich, $M_{\mathrm{w}}=432$ ) as the crosslinking agent (weight ratio of PVP:PMF=2:1) in propylene glycol monomethyl ether acetate (PGMEA). ${ }^{39}$ The PVP solution was spin-coated onto cleaned $\mathrm{SiO}_{2} / \mathrm{Si}$ substrate at $4000 \mathrm{rpm}$ for 40s. The resultant PVP layer was prebaked at $100{ }^{\circ} \mathrm{C}$ for $30 \mathrm{~min}$ and then annealed at $160{ }^{\circ} \mathrm{C}$ for $2 \mathrm{~h}$ in nitrogen atmosphere. Polyacrylonitrile (PAN, Aldrich, $\left.M_{\mathrm{w}}=150,000\right) \mathrm{di}-$ electric layer was spin-coated on $\mathrm{SiO}_{2} / \mathrm{Si}$ substrate from its $\mathrm{N}, \mathrm{N}^{\prime}$-Dimethylformamide (DMF) solution $(10 \mathrm{mg} / \mathrm{ml})$ and then annealed at $150{ }^{\circ} \mathrm{C}$ for $30 \mathrm{~min}$ in nitrogen atmosphere. The thickness of PVA, PVP and PAN film was all $30 \mathrm{~nm}$ and they stored under dry nitrogen prior to usage. For inorganic dielectric layers, $30 \mathrm{~nm} \mathrm{SiO} 2$ and $\mathrm{Si}_{3} \mathrm{~N}_{4}$ were deposited onto cleaned $\mathrm{SiO}_{2} / \mathrm{Si}$ substrate through E-beam evaporation. 5 $\mathrm{nm} \mathrm{Al}$ was deposited onto $\mathrm{SiO}_{2} / \mathrm{Si}$ substrate through thermal evaporation and then treated with oxygen plasma to prepare $\mathrm{Al}_{2} \mathrm{O}_{3}$ dielectric layer.

ODTS Treatment by Liquid Phase Immersion Method. Heavily doped p-type Si wafers with $300 \mathrm{~nm}$ thermally grown silicon oxide were sequentially washed with acetone (three times), and isopropanol (one time) using ultrasonication and dried under flowing nitrogen gas. The $\mathrm{SiO}_{2}$ surface was then cleaned for 1 min under a $500 \mathrm{~W}$ plasma power at $\mathrm{O}_{2}$ flow of $5 \mathrm{sccm}$. Here sccm represents a flow unit of standard-state cubic centimeter per minute. The cleaned silicon substrate was immersed in $3 \mathrm{mM}$ ODTS/toluene solution for $12 \mathrm{~h}$. Toluene solution was purified by distillation prior to usage. The treated samples were sonicated in toluene, acetone, isopropanol and then dried under nitrogen gas.

DDTS/ODTS/ODPA Treatment by Surface Tension Balance Method. For ODTS treatment, firstly, appropriate amount of $200 \mu \mathrm{g} / \mathrm{ml}$ ODTS/hexane solution was dropped on the water surface. After hexane was completely evaporated, add appropriate amount of $50 \mathrm{mg} / \mathrm{ml} \mathrm{PVP/EA} \mathrm{solu-}$ tion on the water surface until the ODTS area cannot be further compressed. ${ }^{40}$ Secondly, $5 \% v / v$ acetone was injected into the water by using a syringe, and then the area of the ODTS can be further compressed by PVP/EA solution slightly. Finally, ODTS monolayer on the water surface was horizontally transferred to arbitrary substrate like Langmuir-Schaefer method and then put it in a closed container containing a little ammonium hydroxide solution (28-30\% in water) or hydrochloric acid solution for $12 \mathrm{~h}$ at room temperature. The substrates were then rinsed with de- ionized water and sonicated in toluene, acetone, isopropanol and then dried under nitrogen gas. Different from ODTS treatment, DDTS/ODPA treatment did not need to add acetone to the water. Besides, the ideal solvent for dispersing ODPA was ethyl acetate, and its optimal concentration was determined to be $12 \mu \mathrm{g} / \mathrm{ml}$.

Fabrication of Various Organic SemiconductorsBased OFETs. Heavily p-doped silicon substrates with a thermally grown $300 \mathrm{~nm}$ silicon dioxide dielectric layer were used as transistor substrates. For bottom-gate topcontact geometry, the organic small-molecular semiconductors (pentacene, CuPc, PTCDI-C8) were deposited on various dielectric layers at a rate of $0.1-0.3 \AA$ /s under a pressure of $3.0 \times 10^{-4} \mathrm{~Pa}$ and a substrate temperature of room temperature to a final thickness of $40 \mathrm{~nm}$ determined by a quartz crystal monitor in the evaporation chamber. The polymer semiconductor (IIDDT-C4) layer was spin-coated on various dielectric layers from its 1,1,2,2-tetrachloroethane (TCE) solution $(4 \mathrm{mg} / \mathrm{ml})$, and then annealed at $175^{\circ} \mathrm{C}$ for $30 \mathrm{~min}$ in the nitrogen atmosphere. ${ }^{41}$ Then, $30 \mathrm{~nm}$ gold source-drain electrodes were deposited on organic semiconductor layers by using shadow masks. Except the OFET device based on the $\mathrm{Al}_{2} \mathrm{O}_{3} / \mathrm{SiO}_{2}$ interface $(W / L=1200 \mu \mathrm{m} / 50$ $\mu \mathrm{m}, W=$ channel width and $L=$ channel length), $W / L$ of other top-contact devices in this study were all 110 ( $L$ of waferscale devices was $120 \mu \mathrm{m}$, and the other was $80 \mu \mathrm{m}$ ). For bottom-gate bottom-contact geometry, source-drain electrodes ( $2 \mathrm{~nm}$ chromium and $30 \mathrm{~nm}$ gold) were patterned by photolithography and lift-off techniques on $\mathrm{SiO}_{2} / \mathrm{Si}$ substrate. The channel width of all devices was $1200 \mu \mathrm{m}$, and channel length was 2-80 $\mu \mathrm{m}$. After deposition of the pentacene or PTCDI-C8, the preparation of bottom-contact OFETs devices were finished.

Fabrication of Flexible OFETs. Flexible bottom-gate top-contact pentacene thin film transistors were fabricated by the following procedures: (1) PET substrate used in the study was successively cleaned with acetone, ethanol, and isopropanol and then dried with flowing nitrogen. Then 30 $\mathrm{nm}$ thickness $\mathrm{Au}$ deposited on the cleaned PET substrate was used to be gate electrode; (2) $200 \mathrm{~nm}$ thickness poly(methyl methacrylate) (PMMA, Aldrich, $M_{\mathrm{w}}=350,000$ ) dielectric layer was spin-coated on $\mathrm{Au} / \mathrm{PET}$ substrate from its ethyl acetate solution (30 mg/ml) and then annealed at $100{ }^{\circ} \mathrm{C}$ for $30 \mathrm{~min}$ in air. Then $300 \mathrm{~nm}$ thickness crosslinked PVP film was spin-coated on PMMA dielectric layer, which was prebaked at $100{ }^{\circ} \mathrm{C}$ for $30 \mathrm{~min}$ and then annealed at 160 ${ }^{\circ} \mathrm{C}$ for $2 \mathrm{~h}$ in nitrogen atmosphere; (3) ODTS monolayer on the water surface was transferred to the surface of PVP/PMMA dielectric layers and then put it in a closed container containing a little ammonium hydroxide solution (28-30\% in water) for $12 \mathrm{~h}$ at room temperature; (4) the substrate was transferred to a vacuum chamber and $40 \mathrm{~nm}$ pentacene was deposited with the deposition rate of 0.1 $\AA / \mathrm{s}$; (5) $30 \mathrm{~nm}$ thickness Au was deposited on the pentacene surface using shadow mask (with the deposition rate of $1.0 \AA / \mathrm{s}$ ) to finish the device construction.

Characterization. Morphology and microstructures of organic semiconductor thin films and different SAM-treated dielectric layers were observed with a Hitachi S4800 fieldemission SEM and a FM-Nanoview LS-AFM (FSMPRECISION, Suzhou, China) in tapping mode. All OFETs were characterized using a Keithley 4200 semiconductor 
characterization system in a nitrogen atmosphere under dark environment. The mobility in the saturated regime was extracted from the following equation: $I_{\mathrm{DS}}=C_{i} \mu(W / 2 L) \times\left(V_{\mathrm{GS}}-V_{\mathrm{T}}\right)^{2}$, where $I_{\mathrm{DS}}$ is the drain current, $C_{i}$ is the capacitance per unit area of the gate dielectric layer, and $V_{\mathrm{GS}}$ and $V_{\mathrm{T}}$ are the gate voltage and threshold voltage, respectively. The capacitance per unit area of the gate dielectric layers is shown in Table S1.

\section{ASSOCIATED CONTENT}

\section{Supporting Information}

The Supporting Information is available free of charge AFM images and OFETs statistical mobilities (PDF)

\section{AUTHOR INFORMATION}

\section{Corresponding Author}

Lei Zhang - School of Chemistry and Chemical Engineering, Nanjing University, Nanjing 210023, China; orcid.org/00000003-1237-3229; Email: zhangleichem@nju.edu.cn

Paolo Samorì-CNRS, ISIS UMR 7006, University of Strasbourg, Strasbourg F-67000, France; orcid.org/0000-0001-6256-8281; Email: samori@unistra.fr

\section{Author}

Baichuan Jiang - School of Chemistry and Chemical Engineering, Nanjing University, Nanjing 210023, China

Yu Che - School of Chemistry and Chemical Engineering, Nanjing University, Nanjing 210023, China

Yurong Chen - School of Chemistry and Chemical Engineering, Nanjing University, Nanjing 210023, China

Yingxuan Zhao - School of Chemistry and Chemical Engineering, Nanjing University, Nanjing 210023, China

Can Wang - CNRS, ISIS UMR 7006, University of Strasbourg, Strasbourg F-67000, France

Wenbin Li - School of Chemistry and Chemical Engineering, Nanjing University, Nanjing 210023, China

Hongxian Zheng - School of Chemistry and Chemical Engineering, Nanjing University, Nanjing 210023, China

Xinxin Huang - School of Chemistry and Chemical Engineering, Nanjing University, Nanjing 210023, China

\section{Author Contributions}

\#B.J. and Y.C. contributed equally to this work.

Notes

The authors declare no competing financial interest.

\section{ACKNOWLEDGMENT}

This work was financially supported by the National Natural Science Foundation (21875100), the NSF of Jiangsu Province (BK20180338), the Fundamental Research Funds for the Central Universities in China (0205/14380222; 0205/14380159), the EC through the ERC project SUPRA2DMAT (GA-833707), the Labex project CSC (ANR-10-LABX-0026 CSC) within the Investissement d'Avenir program ANR-10-IDEX-0002-02, the
International Center for Frontier Research in Chemistry (icFRC) and the Institut Universitaire de France (IUF).

\section{REFERENCES}

(1) Dong R.; Feng X. Making large single crystals of 2D MOFs. Nat. Mater. 2021, 20, 122-123.

(2) Akinwande D.; Huyghebaert C.; Wang C.-H.; Serna M. I.; Goossens S.; Li L.-J.; Wong H. S. P.; Koppens F. H. L. Graphene and twodimensional materials for silicon technology. Nature 2019, 573, 507-518.

(3) Yao J. R.; Zhang Y.; Tian X. Z.; Zhang X. L.; Zhao H. J.; Zhang X. T.; Jie J. S.; Wang X. R.; Li R. J.; Hu W. P. Layer-defining strategy to grow two-dimensional molecular crystals on a liquid surface down to the monolayer limit. Angew. Chem., Int. Ed. 2019, 58, 16082-16086.

(4) Deysher G.; Shuck C. E.; Hantanasirisakul K.; Frey N. C.; Foucher A. C.; Maleski K.; Sarycheva A.; Shenoy V. B.; Stach E. A.; Anasori B.; Gogotsi Y. Synthesis of $\mathrm{Mo}_{4} \mathrm{VAlC}_{4}$ MAX phase and two-dimensional $\mathrm{Mo}_{4} \mathrm{VC}_{4}$ MXene with five atomic layers of transition metals. ACS Nano 2019, 14, 204-217.

(5) Casalini S.; Bortolotti C. A.; Leonardi F.; Biscarini F. Self-assembled monolayers in organic electronics. Chem. Soc. Rev. 2017, 46, 40-71.

(6) Fu B. B.; Wang C.; Sun Y. T.; Yao J. R.; Wang Y.; Ge F. Y.; Yang F. X.; Liu Z. Y.; Dang Y. F.; Zhang X. T.; Shao X. F.; Li R. J.; Hu W. P. A "phase separation" molecular design strategy towards large-area 2D molecular crystals. Adv. Mater. 2019, 31, 1901437.

(7) Tonnelé C.; Champagne B.; Muccioli L.; Castet F. Nonlinear optical contrast in azobenzene-based self-assembled monolayers. Chem. Mater. 2019, 31, 6759-6769.

(8) Salinas M.; Jaeger C. M.; Amin A. Y.; Dral P. O.; Meyer-Friedrichsen T.; Hirsch A.; Clark T.; Halik M. The relationship between threshold voltage and dipolar character of self-assembled monolayers in organic thin-film transistors. J. Am. Chem. Soc. 2012, 134, 12648-12652.

(9) Ligorio G.; Cotella G. F.; Bonasera A.; Zorn Morales N.; Carnicella G.; Kobin B.; Wang Q.; Koch N.; Hecht S.; List-Kratochvil E. J. W.; Cacialli F. Modulating the luminance of organic light-emitting diodes via optical stimulation of a photochromic molecular monolayer at transparent oxide electrode. Nanoscale 2020, 12, 5444-5451.

(10) Han B.; Li Y.; Ji X.; Song X. N.; Ding S. S.; Li B. L.; Khalid H.; Zhang Y. G.; Xu X. N.; Tian L. X.; Dong H. L.; Yu X.; Hu W. P. Systematic modulation of charge transport in molecular devices through facile control of molecule-electrode coupling using a double self-assembled monolayer nanowire junction. J. Am. Chem. Soc. 2020, 142, 97089717.

(11) Lee J.; Chang H.; Kim S.; Bang G. S.; Lee H. Molecular monolayer nonvolatile memory with tunable molecules. Angew. Chem., Int. Ed. 2009, 48, 8501-8504.

(12) Shi Y. J.; Jiang L.; Liu J.; Tu Z. Y.; Hu Y. Y.; Wu Q. H.; Yi Y. P.; Gann E.; McNeill C. R.; Li H. X.; Hu W. P.; Zhu D. B.; Sirringhaus H. Bottomup growth of n-type monolayer molecular crystals on polymeric substrate for optoelectronic device applications. Nat. Commun. 2018, 9, 2933.

(13) Sizov A. S.; Agina E. V.; Ponomarenko S. A. Self-assembled interface monolayers for organic and hybrid electronics. Russ. Chem. Rev. 2019, 88, 1220-1247.

(14) Lee C.-H.; Lee G.-H.; van der Zande A. M.; Chen W.; Li Y.; Han M.; Cui X.; Arefe G.; Nuckolls C.; Heinz T. F.; Guo J.; Hone J.; Kim P. Atomically thin $\mathrm{p}-\mathrm{n}$ junctions with van der Waals heterointerfaces. Nat. Nanotechnol. 2014, 9, 676-681.

(15) Gobbi M.; Orgiu E.; Samorì P. When 2D materials meet molecules: Opportunities and challenges of hybrid organic/inorganic van der Waals heterostructures. Adv. Mater. 2018, 30, 1706103.

(16) Zhang T.; Qi H.; Liao Z.; Horev Y. D.; Panes-Ruiz L. A.; St Petkovf P.; Zhang Z.; Shivhare R.; Zhang P.; Liu K.; Bezugly V.; Liu S.; Zheng Z.; Mannsfeld S.; Heine T.; Cuniberti G.; Haick H.; Zschech E.; Kaiser U.; Dong R.; Feng X. Engineering crystalline quasi-two-dimensional polyaniline thin film with enhanced electrical and chemiresistive sensing performances. Nat. Commun. 2019, 10, 4225. 
(17) Li M.; Mangalore D. K.; Zhao J.; Carpenter J. H.; Yan H.; Ade H.; Yan H.; Mullen K.; Blom P. W. M.; Pisula W.; de Leeuw D. M.; Asadi K. Integrated circuits based on conjugated polymer monolayer. Nat. Commun. 2018, 9, 451.

(18) Jin Y. H.; Hu Y. M.; Ortiz M.; Huang S. F.; Ge Y. Q.; Zhang W. Confined growth of ordered organic frameworks at an interface. Chem. Soc. Rev. 2020, 49, 4637-4666.

(19) Walter S. R.; Youn J.; Emery J. D.; Kewalramani S.; Hennek J. W.; Bedzyk M. J.; Facchetti A.; Marks T. J.; Geiger F. M. In-situ probe of gate dielectric-semiconductor interfacial order in organic transistors: origin and control of large performance sensitivities. J. Am. Chem. Soc. 2012, 134, 11726-11733.

(20) Nakano M.; Osaka I.; Takimiya K. Control of major carriers in an ambipolar polymer semiconductor by self-assembled monolayers. Adv. Mater. 2017, 29, 1602893.

(21) Mityashin A.; Roscioni O. M.; Muccioli L.; Zannoni C.; Geskin V.; Cornil J.; Janssen D.; Steudel S.; Genoe J.; Heremans P. Multiscale modeling of the electrostatic impact of self-assembled monolayers used as gate dielectric treatment in organic thin-film transistors. ACS Appl. Mater. Interfaces 2014, 6, 15372-15378.

(22) Vilan A.; Aswal D.; Cahen D. Large-area, ensemble molecular electronics: Motivation and challenges. Chem. Rev. 2017, 117, 4248-4286.

(23) Chen H.; Zhang W.; Li M.; He G.; Guo X. Interface engineering in organic field-effect transistors: Principles, applications, and perspectives. Chem. Rev. 2020, 120, 2879-2949.

(24) Ito Y.; Virkar A. A.; Mannsfeld S.; Oh J. H.; Toney M.; Locklin J.; Bao Z. Crystalline ultrasmooth self-assembled monolayers of alkylsilanes for organic field-effect transistors. J. Am. Chem. Soc. 2009, 131, 9396-9404.

(25) Li H.; Shi Y.; Han G.; Liu J.; Zhang J.; Li C.; Liu J.; Yi Y.; Li T.; Gao X.; Di C.; Huang J.; Che Y.; Wang D.; Hu W.; Liu Y.; Jiang L. Monolayer two-dimensional molecular crystals for an ultrasensitive OFETbased chemical sensor. Angew. Chem., Int. Ed. 2020, 59, 43804384 .

(26) Jiang L.; Dong H. L.; Meng Q.; Li H.; He M.; Wei Z.; He Y.; Hu W. P. Millimeter-sized molecular monolayer two-dimensional crystals. Adv. Mater. 2011, 23, 2059-2063.

(27) Xu G.; Bao Z.; Groves J. T. Langmuir-Blodgett films of regioregular poly(3-hexylthiophene) as field-effect transistors. Langmuir 2000, 16, 1834-1841.

(28) Ariga K. Don't forget Langmuir-Blodgett films 2020: Interfacial nanoarchitectonics with molecules, materials, and living objects. Langmuir 2020, 36, 7158-7180.

(29) Virkar A.; Mannsfeld S.; Oh J. H.; Toney M. F.; Tan Y. H.; Liu G. y.; Scott J. C.; Miller R.; Bao Z. The role of OTS density on pentacene and $\mathrm{C} 60$ nucleation, thin film growth, and transistor performance. Adv. Funct. Mater. 2009, 19, 1962-1970.
(30) Sizov A. S.; Trul A. A.; Chekusova V.; Borshchev O. V.; Vasiliev A. A.; Agina E. V.; Ponomarenko S. A. Highly Sensitive Air-Stable Easily Processable Gas Sensors Based on Langmuir-Schaefer Monolayer Organic Field-Effect Transistors for Multiparametric $\mathrm{H}_{2} \mathrm{~S}$ and $\mathrm{NH}_{3}$ Real-Time Detection. ACS Appl. Mater. Interfaces 2018, 10 , 43831-43841.

(31) Chen H.; Guo X. Unique role of self-assembled monolayers in carbon nanomaterial-based field-effect transistors. Small 2013, 9, 1144-1159.

(32) Kang D. H.; Shim J.; Jang S. K.; Jeon J.; Jeon M. H.; Yeom G. Y.; Jung W. S.; Jang Y. H.; Lee S.; Park J. H. Controllable nondegenerate p-type doping of tungsten diselenide by octadecyltrichlorosilane. ACS Nano 2015, 9, 1099-1107.

(33) Kobayashi S.; Nishikawa T.; Takenobu T.; Mori S.; Shimoda T.; Mitani T.; Shimotani H.; Yoshimoto N.; Ogawa S.; Iwasa Y. Control of carrier density by self-assembled monolayers in organic fieldeffect transistors. Nat. Mater. 2004, 3, 317-322.

(34) Kim J.; Kang B.; Cho K. Heat-assisted photoacidic oxidation method for tailoring the surface chemistry of polymer dielectrics for low-power organic soft electronics. Adv. Funct. Mater. 2019, 29, 1806030.

(35) Xu J.; Wang S. H.; Wang G. J. N.; Zhu C. X.; Luo S. C.; Jin L. H.; Gu X. D.; Chen S. C.; Feig V. R.; To J. W. F.; Rondeau-Gagne S.; Park J.; Schroeder B. C.; Lu C.; Oh J. Y.; Wang Y. M.; Kim Y. H.; Yan H.; Sinclair R.; Zhou D. S.; Xue G.; Murmann B.; Linder C.; Cai W.; Tok J. B. H.; Chung J. W.; Bao Z. N. Highly stretchable polymer semiconductor films through the nanoconfinement effect. Science 2017, 355, 5964.

(36) Xiao M.; Liu J.; Hu Y.; Wang S.; Jiang L. Nonideal double-slope effect in organic field-effect transistors. Front. Phys. 2021, 16, 13305.

(37) Yao Y.; Zhang L.; Leydecker T.; Samorì P. Direct photolithography on molecular crystals for high performance organic optoelectronic devices. J. Am. Chem. Soc. 2018, 140, 6984-6990.

(38) Lamping S.; Buten C.; Ravoo B. J. Functionalization and patterning of self-assembled monolayers and polymer brushes using microcontact chemistry. Acc. Chem. Res. 2019, 52, 1336-1346.

(39) Zhang L.; Pasthukova N.; Yao Y.; Zhong X.; Pavlica E.; Bratina G.; Orgiu E.; Samorì P. Self-Suspended Nanomesh Scaffold for Ultrafast Flexible Photodetectors Based on Organic Semiconducting Crystals. Adv. Mater. 2018, 30, 1801181.

(40) Zhang L.; Zhong X.; Pavlica E.; Li S.; Klekachev A.; Bratina G.; Ebbesen T. W.; Orgiu E.; Samorì P. A Nanomesh Scaffold for Supramolecular Nanowire Optoelectronic Devices. Nat. Nanotechnol. 2016, 11, 900-906.

(41) Lei T.; Dou J.-H.; Pei J. Influence of Alkyl Chain Branching Positions on the Hole Mobilities of Polymer Thin-Film Transistors. Adv. Mater. 2012, 24, 6457-6461. 